\title{
Oft auch ohne Medikamente nicht fahrtüchtig
}

Fragestellung: Besteht die Fahrtüchtigkeit im Straßenverkehr bei Patienten mit Schizophrenie, die keine Medikamente einnehmen?

Hintergrund: Schizophrene Patienten zeigen oft Störungen von Aufmerksamkeit, Gedächtnis, Exekutivfunktionen sowie psychomotorischen Fähigkeiten. Aufgrund der meist sedierenden Effekte könnte die Medikation großen Einfluss auf die Fahrtüchtigkeit haben. Daten zum Effekt von nicht pharmakologisch therapierten Erkrankten liegen kaum vor.

Patienten und Methodik: Bei 26 Patienten mit Schizophrenie (13 ersterkrankt, 13 mit früheren Episoden) und 20 gesunden Probanden, alle im Alter von zirka 30 Jahren, wurden unverblindet mithilfe eines Computertests die Verarbeitungsgeschwindigkeit verkehrstypischer visueller Reize, Reaktionsgeschwindigkeit, Stresstoleranz, selektive Aufmerksamkeit, Konzentration und Vigilanz bei monotonen Tätigkeiten untersucht. Als fahruntüchtig galten Personen, die mehr als eine Standardabweichung unter dem Durchschnitt eines Kollektivs von Autofahrern lagen. Dieser Test zeigte in der Vergangenheit eine Genauigkeit von über $80 \%$ und entspricht deutschen Richtlinien. Zudem wurden der PANSS-Fragebogen zur Symptomschwere der Schizophrenie und demografisch-klinische Daten erhoben.

Segmiller FM, Buschert V, Laux $\mathrm{G}$ et al. Driving skills in unmedicated first and recurrent episode schizophrenic patients. Eur Arch Psychiatry Clin Neurosci 2017; 267: $83-8$
Ergebnisse: Alle Analysen erfolgten alterskontrolliert. Elf von 23 Patienten $(\sim 42 \%)$ und alle 20 gesunden Probanden wurden als fahrsicher eingestuft. Bei $32 \%$ der Patienten war die Fahrtüch-

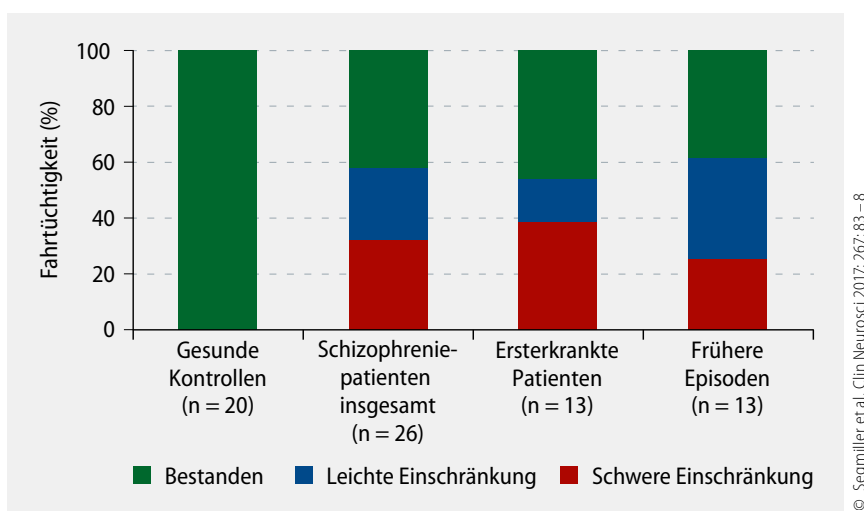

1 Fahrtüchtigkeit bei Patienten mit Schizophrenie und Gesunden.

tigkeit schwer und bei $26 \%$ leicht eingeschränkt. Die Fahrtüchtigkeit war statistisch signifikant schlechter bei Ersterkrankten (38\% schwere Einschränkungen) als bei Patienten mit früheren Episoden (25\% schwere Einschränkungen) ( Abb. 1). Beide Patientengruppen waren signifikant schlechter als die Kontrollgruppe. In allen einzelnen Funktionstests schnitten die Patienten signifikant schlechter ab als die Gesunden. Ersterkrankte waren gegenüber der Kontrollgruppe weniger stresstolerant und Patienten mit früheren Episoden zeigten gegenüber der Kontrollgruppe weniger Vigilanz bei monotonen Aufgaben. Einschränkungen in den Funktionstests korrelierten nicht signifikant mit der PANSS-Symptomschwere der Schizophrenie.

Schlussfolgerung: Die Fahrtüchtigkeit von Patienten mit Schizophrenie ist oft auch ohne Medikation eingeschränkt.

\section{- Kommentar von Friedrich Duge und Nadine Dreimüller, Mainz}

\section{Wichtige Fragen zur Fahrtüchtigkeit offen}

Wie unterscheidet sich die Fahrtüchtigkeit von Patienten mit Schizophrenie von denen mit anderen psychiatrischen Erkrankungen? Wieso gab es keine signifikante Korrelation zwischen Symptomschwere und Fahrtüchtigkeit? Ist die Fahrtüchtigkeit eventuell auch außerhalb der Episode eingeschränkt? Wie ist die Auswirkung der medikamentösen Behandlung? Diese und viele weitere Fragen lässt die Arbeit unbeantwortet. Dies sollte man jedoch nicht der Publikation als Schwäche anlasten, sondern zeigt eher, dass wichtige und insbesondere versorgungsrelevante Daten in diesem Bereich fehlen.

Die Arbeit selbst leidet unter den typischen Schwächen einer Beobachtungsstudie in einem wenig untersuchten Gebiet: keine Verblindung, kleine Fallzahl und kein experimentell-randomisiertes Design. Die Arbeit sollte also dringend durch weitere Untersuchungen ergänzt werden. Fahrtüchtigkeit ist ein Thema von hoher praktischer Relevanz für Patienten und ihre
Behandler und der Mangel an Untersuchungen zu diesem Thema ist bedenkenswert. Die Arbeit stößt wichtige Forschungswege an und liefert dabei eine zumindest vorläufige Datenbasis mit. Insgesamt eine sehr wichtige Untersuchung bei der auf baldige Folgeergebnisse zu hoffen ist.

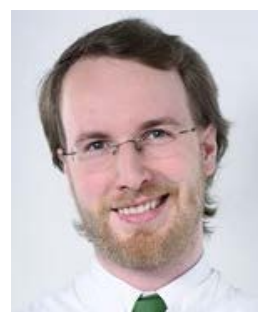

Friedrich Duge und Dr. med. Nadine Dreimüller, Mainz

Klinik für Psychiatrie und Psychotherapie, Universitätsmedizin Mainz E-Mail: friedrich.duge@unimedizin-mainz.de; nadine.dreimueller@unimedizin-mainz.de 